\title{
Diagnosis and Management of Depression in CRS: A Knowledge, Attitudes and Practices Survey
}

\author{
Amarbir S. Gill ${ }^{1}$ Joshua M. Levy ${ }^{2}$ Machelle Wilson ${ }^{3}$ \\ ${ }^{1}$ Department of Otolaryngology - Head and Neck Surgery, University of \\ California, Davis School of Medicine, Sacramento, California, United States \\ 2 Emory University Department of Otolaryngology - Head and Neck \\ Surgery, Atlanta, GA \\ ${ }^{3}$ Department of Public Health Sciences/Biostatistics, Clinical and \\ Translational Science Center, University of California Davis, \\ Sacramento, CA \\ ${ }^{4}$ VA Northern California Healthcare System, Sacramento, CA \\ Int Arch Otorhinolaryngol 2021;25(1):e48-e53.
}

E. Bradley Strong ${ }^{1}$ Toby O. Steele $\mathrm{e}^{1,4}$

\begin{abstract}
Address for correspondence Toby Steele, MD, Department of Otolaryngology - Head and Neck Surgery, University of California, Davis School of Medicine, 2521 Stockton Boulevard, Sacramento, CA 95817, United States (e-mail: tosteele@ucdavis.edu).
\end{abstract}

\begin{abstract}
Keywords

- depression

- chronic rhinosinusitis

- screen

- diagnosis

- management

- quality of life
\end{abstract}

Introduction Comorbid major depressive disorder (MDD) is present in up to $25 \%$ of chronic rhinosinusitis (CRS) cases and provides prognostic information for patients undergoing endoscopic sinus surgery (ESS). Clinical visits offer an opportunity to identify at-risk patients. Objective The purpose of the present study is to evaluate practice patterns among members of the American Rhinologic Society (ARS) in screening for/diagnosing MDD. Methods A 21-question survey was distributed to 1,206 members of the ARS from May 26, 2018 to June 12, 2018. The impact of demographic factors, including hospital setting, fellowship status, and experience were assessed through chi-squared analysis. Results A total of 80 members of the ARS completed the survey, yielding a response rate of $7 \%$. Half of the respondents worked in academic settings and $43 \%$ had completed a rhinology fellowship. Twenty percent of the participants felt comfortable diagnosing or managing MDD, while only $10 \%$ of participants screened for MDD in patients with CRS. Respondents cited a lack of training (76\%) and unfamiliarity with diagnostic criteria (76\%) as barriers to the routine assessment of MDD. Most respondents (95\%) considered comorbid psychiatric illness to negatively impact outcomes following ESS. Fellowship-trained respondents were significantly more likely to implement screening tools in their practice $(p=0.05)$, and believe in the negative impact of MDD on postoperative outcomes $(p=0.007)$, cost of care $(p=0.04)$ and quality of life $(p=0.047)$.

Conclusion Amongst ARS members, $95 \%$ of the respondents consider comorbid MDD to negatively impact patient outcomes following ESS. Regardless, a large proportion of surgeons neither screen nor feel comfortable diagnosing MDD.

\section{Introduction}

Comorbid major depressive disorder (MDD) has been shown to be present in up to $40 \%$ of patients with chronic rhinosinusitis (CRS). ${ }^{1-6}$ Several studies demonstrate an increased prevalence of mood disorder symptoms (i.e., depression) in patients with CRS compared with controls, particularly patients with a diagnosis of CRS without nasal polyposis (CRSsNP). ${ }^{7,8}$ Moreover, there may be a disease-dependent relationship between the severity of sinus symptoms and the prevalence of received

June 22, 2019

accepted

December 3, 2019

published online

April 24, 2020
DOI https://doi.org/ $10.1055 / \mathrm{s}-0040-1701268$ ISSN 1809-9777.

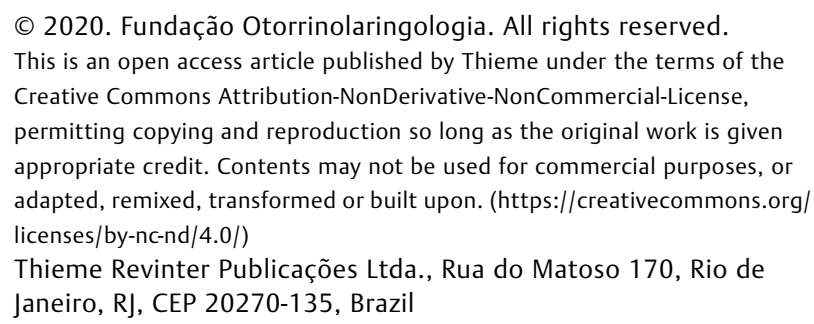


concomitant mood disorder, as patients with advanced CRS are significantly more likely to have severe depression and/or anxiety compared with patients with less advanced disease. ${ }^{9}$

Patients with CRS and MDD have increased severity of both sinonasal symptoms (facial pain, nasal discharge, and nasal obstruction) as well as extra-rhinologic symptoms, such as loss of productivity. Indeed, CRS patients with comorbid MDD have greater preoperative impairment in disease-specific quality of life (QOL), as measured by the SNOT-22 questionnaire, when compared with CRS patients without MDD. ${ }^{10}$ Although their QOL shows similar improvement with endoscopic sinus surgery (ESS), their postoperative SNOT-22 scores remain lower than their counterparts with no history of MDD. ${ }^{2}$ In other words, CRS patients with MDD start with greater impairments in QOL before surgery and finish with greater impairments in QOL after surgery.

Identification of comorbid MDD provides clinically relevant prognostic information for CRS patients undergoing medical or surgical therapies. Otolaryngology clinic visits provide an opportunity to identify at-risk patients who may then be appropriately treated or referred for further evaluation. ${ }^{11}$ The most commonly employed depressive disorder screening tools include the Patient Health Questionnaire -9 (PHQ-9), Patient Health Questionnaire-2 (PHQ-2), Beck Depression Inventory (BDI), and Hospital Anxiety and Depression Scale (HADS). The goal of the present study is to evaluate practice patterns of community and university-based otolaryngologists in the diagnosis and management of MDD in patients with chronic rhinosinusitis.

\section{Methods}

A 21-question survey (-Appendix A - Available online) was created by a multi-disciplinary, multi-institutional team comprised of the following experts: biostatistician, family practice physician familiar with the diagnosis and management of mood disorders, as well as a rhinology team at an academic tertiary care center. This survey was distributed to 1,206 members of the American Rhinologic Society (ARS) from May 26, 2018 to June 12,2018 . The survey goals were to establish the demographic data of respondents, identify screening and management patterns of MDD for chronic rhinosinusitis patients, and query respondents on the impact of MDD in the CRS patient population. The following demographic data were collected: fellowship training, clinical experience (years in practice), hospital setting (academic versus non-academic), and geographic location of the respondents (Northeast, Midwest, South and West) (-Fig. 1).

Major depressive disorder screening and management patterns were assessed based on the following categories: 1) Comfort level and training in routine screening for MDD, 2) management strategies for MDD (i.e., primary care, psychiatry or psychology referrals, detailed psychiatric history, laboratory analysis, or the initiation of pharmacotherapies). Members of the ARS were asked about the prevalence of empty nose syndrome (ENS) within their practice, and their opinion on the impact of psychiatric illness on the outcomes of ESS. The impact of MDD was assessed based on participant responses to

\section{Respondents}

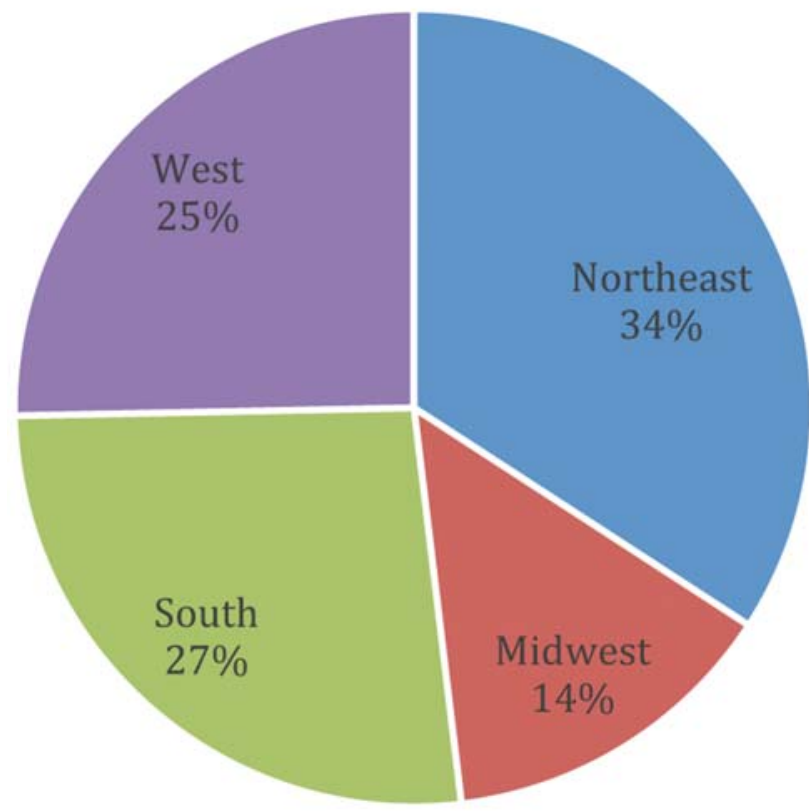

Fig. 1 Respondents divided by geographic region within the USA.

the following statements: MDD negatively impacts QOL, MDD negatively impacts cost of care, MDD negatively impacts postoperative outcomes, or MDD does not have a negative impact.

Further subgroup analysis was performed via chi-squared testing to analyze the independent impact and association of each demographic factor on survey responses. The threshold for significance was set at $p<0.05$. Statistical analysis was supported by the National Center for Advancing Translational Sciences, National Institutes of Health, through grant number UL1 TR001860. The content is solely the responsibility of the authors and does not necessarily represent the official views of the NIH.

\section{Results}

There were 80 members of the ARS who completed the survey, yielding a response rate of $7 \%$. The survey responses were evenly spread out across the major geographic areas of the USA, with the majority emanating from the Northeast region (34\%) (-Fig. 1).

\section{Training and Clinical Practice}

Half of the respondents worked in an academic setting $(n=40)$ and had been in practice longer than 16 years $(51 \%, n=41)$. Less than half $(43 \%)$ of the respondents completed a formal rhinology fellowship and only $4 \%$ claimed to have formal training in the assessment and management of MDD (-Table 1).

\section{MDD Screening Practices}

Although the majority (95\%) of participants believed that comorbid psychiatric illness negatively impacts outcomes following ESS, only $10 \%$ of respondents screened for MDD in patients with CRS (-Table 1). Specifically, 94\% believed that 
Table 1 Demographic information for practice patterns in screening and managing major depressive disorder among otolaryngologists

\begin{tabular}{|c|c|}
\hline Survey Question & Respondents (\%) \\
\hline Hospital setting & $80(100)$ \\
\hline Academic & $40(50)$ \\
\hline Private & $24(30)$ \\
\hline Joint academic/private & $8(10)$ \\
\hline Hospital employee & $8(10)$ \\
\hline Fellowship trained & $80(100)$ \\
\hline Yes & $34(43)$ \\
\hline No & $46(57)$ \\
\hline Years in practice & $80(100)$ \\
\hline $0-5$ & $18(23)$ \\
\hline $6-10$ & $11(14)$ \\
\hline $11-15$ & $10(12)$ \\
\hline$>16$ & $41(51)$ \\
\hline \multicolumn{2}{|l|}{ Routine MDD screening } \\
\hline Yes & $10 \%$ \\
\hline No & $90 \%$ \\
\hline Formal training MDD management & $80(100)$ \\
\hline Yes & $3(4)$ \\
\hline No & $77(96)$ \\
\hline \multicolumn{2}{|l|}{$\begin{array}{l}\text { Comfortable screening/ } \\
\text { managing MDD }\end{array}$} \\
\hline Comfortable/very comfortable & $20 \%$ \\
\hline Neutral & $34 \%$ \\
\hline Uncomfortable/very uncomfortable & $46 \%$ \\
\hline \multicolumn{2}{|l|}{ Management options for at risk of MDD } \\
\hline PCP referral & $83 \%$ \\
\hline Medication & $8 \%$ \\
\hline Psychiatry referral & $54 \%$ \\
\hline Psychology referral & $44 \%$ \\
\hline Order laboratories & $17 \%$ \\
\hline Detailed psychiatric history & $17 \%$ \\
\hline $\begin{array}{l}\text { Psychiatric illness impacts } \\
\text { ESS outcomes }\end{array}$ & $95 \%$ \\
\hline Negatively impacts QOL & $94 \%$ \\
\hline Negatively impacts cost of care & $40 \%$ \\
\hline $\begin{array}{l}\text { Negatively impacts } \\
\text { post-operative outcomes }\end{array}$ & $38 \%$ \\
\hline \multicolumn{2}{|l|}{ Treat ENS in your practice } \\
\hline Yes & $59 \%$ \\
\hline No & $41 \%$ \\
\hline \multicolumn{2}{|l|}{$\begin{array}{l}\text { Believe ENS is a manifestation of } \\
\text { psychiatric illness }\end{array}$} \\
\hline Yes & $59 \%$ \\
\hline No & $41 \%$ \\
\hline
\end{tabular}

Abbreviations: ENS, empty nose syndrome; ESS, endoscopic sinus surgery; MDD, major depressive disorder; $\mathrm{PCP}$, primary care physician; QOL, quality of life. the postoperative QOL to be negatively affected in the setting of concomitant psychiatric illness, $40 \%$ reported cost of care to be negatively affected, and $38 \%$ of respondents reported postoperative outcomes to be negatively affected in the setting of concomitant psychiatric illness. The majority of respondents (51\%) counseled patients about expected post-operative outcomes for CRS patients with comorbid MDD.

\section{Major Depressive Disorder Management Practices}

Twenty percent of providers were comfortable confirming the diagnosis of MDD or managing it in those identified to be at risk on screening. Participants cited a lack of training (35\%) and unfamiliarity with diagnostic criteria (41\%) as the most common reasons for discomfort in establishing a diagnosis of MDD. In the $10 \%$ of respondents who screened for MDD $(n=8), 83 \%$ managed at-risk patients by referring the patient to their primary care physician for treatment, while referral to a psychologist or psychiatrist was less common (44\% and $54 \%$, respectively). A detailed psychiatric history and additional laboratory work were obtained by $17 \%$ of screeners. Eight percent of screeners reported comfort prescribing medication for management.

\section{Subgroup Analysis}

A chi-squared analysis was performed to analyze the impact of demographic factors (i.e., geography, hospital setting, practice type, fellowship status, MDD training, and clinical experience) on response data (-Table 2). Although the majority of respondents did not feel comfortable screening for MDD, fellowship-trained individuals were significantly more likely to implement screening tools in their practice $(p=0.05)$ compared with those who were not fellowship trained. When respondents did screen for depression, they were more likely to select the PHQ-9 questionnaire compared with PHQ-2, BDI, and clinical history and physical exam $(p=0.003)$. Fellowship trained otolaryngologists were significantly more likely to consider the history and physical exam as a sufficient screening tool for $\operatorname{MDD}(p=0.017)$. The 3 individuals who were formally trained in MDD evaluation also felt a good history and physical exam to be the best means to identify those at risk of MDD.

Respondents working in an academic setting or with selfreported fellowship training were significantly more likely to believe in the negative impact of MDD on postoperative outcomes following sinus surgery $(p=0.05$ and $p=0.007$, respectively). Fellowship-trained respondents were more likely to believe that MDD also negatively affected the cost of care $(p=0.04)$ and QOL $(p=0.047)$. Otolaryngologists working in academic setting were significantly more likely to see patients with diagnosis of ENS $(p=0.05)$.

\section{Discussion}

There is a high prevalence of comorbid psychiatric illness in patients with chronic rhinosinusitis. Zhou et al queried the National Health Interview Survey for 19 million patients with CRS and noted that $22 \%$ of the patients had a prior diagnosis of depressive symptoms. After multivariate 
Table 2 Subgroup analysis: the effect of demographics on practice patterns

\begin{tabular}{|l|l|l|l|l|l|l|}
\hline $\mathbf{N}=\mathbf{8 0}$ & & Demographics & & & \\
\hline Practice Pattern & Region & Academic hospital & Fellowship trained & Clinical experience & MDD trained & All \\
\hline Screening tool & $p<0.05$ & $p<0.05$ & $p<0.05$ & $p<0.05$ & $p<0.05$ & $p<0.05$ \\
\hline PHQ 9 & $p<0.05$ & $p<0.05$ & $p<0.05$ & $p<0.05$ & $p<0.05$ & $\mathbf{0 . 0 0 3}$ \\
\hline PHQ 2 & $p<0.05$ & $p<0.05$ & $p<0.05$ & $p<0.05$ & $p<0.05$ & $p<0.05$ \\
\hline Becks & $p<0.05$ & $p<0.05$ & $p<0.05$ & $p<0.05$ & $p<0.05$ & $p<0.05$ \\
\hline H\&P & $p<0.05$ & $p<0.05$ & $\mathbf{0 . 0 1 7}$ & $p<0.05$ & $\mathbf{0 . 0 2 1 7}$ & $p<0.05$ \\
\hline Decreases QOL & $p<0.05$ & $\mathbf{0 . 0 4 7}$ & $p<0.05$ & $p<0.05$ & $p<0.05$ & $p<0.05$ \\
\hline Increases cost & $p<0.05$ & $\mathbf{0 . 0 4 2}$ & $p<0.05$ & $p<0.05$ & $p<0.05$ & $p<0.05$ \\
\hline Increases complications & $p<0.05$ & $\mathbf{0 . 0 0 7}$ & $\mathbf{0 . 0 5}$ & $p<0.05$ & $p<0.05$ & $p<0.05$ \\
\hline Routine screening & $p<0.05$ & $p<0.05$ & $\mathbf{0 . 0 5}$ & $p<0.05$ & $p<0.05$ & $p<0.05$ \\
\hline Prevalence of ENS & $p<0.05$ & $\mathbf{0 . 0 5}$ & $p<0.05$ \\
\hline
\end{tabular}

Abbreviations: ENS, empty nose syndrome; H\&P, history and physical; QOL, quality of life.

regression analysis, the prevalence of sinusitis was higher in patients with depressive symptoms. ${ }^{1}$ The authors advocated for targeting interventions among these patients with comorbidities associated with sinus disease. Schlosser et al performed a comprehensive systematic review examining the prevalence of MDD among patients with CRS. Although it varied slightly depending on the method of diagnosis (i.e., physician diagnosed, PHQ HADS, BID), the overall frequency was significant, ranging from 18 to $40 \%$ of patients with CRS. ${ }^{4}$ Given this opportunity to improve care for patients with increased risk of comorbid psychiatric illness, we sought to determine the knowledge, attitudes and practices of ARS members in the evaluation and management of MDD in patients with chronic rhinosinusitis. The ARS was chosen as a representative body of otolaryngologists who have a clinical emphasis or interest in the care of patients with rhinologic diseases. We further sought to identify evaluation strategies currently being implemented in otolaryngology clinics to screen or diagnose MDD as well as determine which treatment modalities are being offered.

The data demonstrate agreement amongst most study respondents that psychiatric illness negatively impacts outcomes of sinus surgery. Nevertheless, few individuals screen for mental illness in the CRS population. The paucity of screening tools implemented in rhinology clinics is not unique to otolaryngology surgeons. Rather, prior survey studies assessing medical and surgical provider practice patterns have documented a strong belief that MDD negatively impacts patient/ surgical outcomes, but routine screening for depression appears limited. ${ }^{12,13}$ Young et al examined compliance of spine surgeons with the United States Preventive Services Task Force recommendation that all patients considered for spine surgery be screened for mood symptoms using the Presurgical Psychological Screening. ${ }^{12}$ Although a large proportion of the surgeon respondents agreed that psychosocial factors negatively impact patient outcomes, including pain relief $(81 \%)$ and return to work (83\%), only $37 \%$ of respondents were compliant with routine screening.
Furthermore, Goldin Evans et al performed a systematic review of practice patterns of primary care physicians, pediatricians, and gynecologists regarding routine postpartum depression screening in new mothers. ${ }^{13}$ The authors examined 11 articles and noted that $55 \%$ of the respondents reported performing a "symptom check" to assess for depression, whereas 30\% stated they never evaluated for depression in this patient population. When asked about barriers to screening, two-thirds of the specialists cited lack of time, with an additional one-third citing lack of training or knowledge. The responses in the study by Goldin Evans parallel the responses seen amongst ARS respondents, in which $40 \%$ of the respondents stated they were not comfortable screening for depression because further treatment/evaluation strategies were unclear if the screen were positive. Only three ARS surgeon respondents had any formal training in the evaluation of MDD. Otolaryngology respondents may feel that even if they are performing the screen, there is no guidance on the next best action (i.e., how to confirm the diagnosis or the next steps in the management of MDD).

To further explore this clinical challenge, ARS members were asked the following questions: if given the choice, how would you intervene on patients that were identified to be atrisk of MDD? The vast majority of respondents (83\%) stated they would refer these patients to the primary care physician for further management. Goldin Evans et al also asked a similar question of their cohort, and noted that up to $80 \%$ of OB/GYN and pediatric specialists replied that they would refer patients to a mental health provider, whereas $70 \%$ of primary care physicians stated they would treat identified post partum depression (PPD) themselves. ${ }^{13}$ This highlights varying comfort levels and formal training between specialties in assessing and managing MDD. This is important to note as it can help personalize screening and referral strategies based on specialty emphasis.

Another reason otolaryngologists may not screen for MDD may be the lack of data supporting a positive impact on outcomes after ESS. Although no study has analyzed this relationship to date, three studies have examined whether 
medical or surgical treatment of sinus disease can affect underlying MDD/anxiety. ${ }^{2,5,14}$ Schlosser et al prospectively examined 685 patients with CRS and noted that 24\% were noted to have depression based on the PHQ- 2 score. ${ }^{5}$ With medical and/or surgical intervention, the patient cohort demonstrated significant improvement in their mean PHQ-2 scores. Of the 167 patients initially noted to have depression, $65 \%$ had resolution of their MDD diagnosis with management of the underlying CRS. Similarly, Litvack et al examined a cohort of 76 CRS patients, of whom 25\% screened positive for depression; the authors demonstrated significant improvement in severity of depression in this cohort $(p=0.017)$ after ESS. $^{2}$ In contrast to the Litvack and Schlosser studies, Adams et al demonstrated that despite improvement in diseasespecific QOL, 44 CRS patients treated with ESS had no improvement in their depression or anxiety as measured by their HADS score postoperatively. ${ }^{14}$ Thus, the effect of ESS on underlying depression remains unclear.

Considering that the direct effect of screening on disease outcome has not been well examined among any medical specialty in the literature, a future study represents a muchneeded future direction given the significant mental health burden in the CRS patient population. Although Schlosser, Litvack, and Adams may not have demonstrated a reproducible effect of ESS on preexisting mental health conditions, it is possible that improving underlying MDD/anxiety prior to ESS can have an impact of the degree of QOL improvement in sinus symptoms. ${ }^{2,4,14}$ For example, a recent prospective study by Banoub et al examined 610 patients with CRS and suggested that there is a threshold limit on the degree of quality of life impairment attributable purely to sinus symptom burden as assessed by SNOT$22 .{ }^{15}$ Further deficiency in QOL was purely associated with presence of depression as detected by PHQ-2. Thus, it is possible that by modulating the underlying depression we can positively affect the QOL.

Although some physicians consider a good psychiatric clinical history and exam to be the best tool to identify those at risk of mental disorders, time constraints and the focused clinical history taking during an otolaryngology clinic visit may limit the ability of the clinician to thoroughly perform a psychiatric history and exam. These data demonstrate that ARS respondents are reluctant to take detailed psychiatric histories (3\% of respondents) and few are familiar with the Diagnostic and Statistical Manual of Mental Disorders (DSM) diagnostic criteria. One such alternative to a detailed psychiatric history for the screening of comorbid psychiatric illness is a mood symptom questionnaire during their initial visit paperwork, such as the PHQ-9, which was the most commonly cited questionnaire in the present study. This questionnaire has undergone rigorous validation against gold standard DSM IV criteria for MDD. Gillbody et al conducted a recent diagnostic meta-analysis examining 17 separate validation studies across multiple specialties, including otolaryngology. The authors concluded that the PQH-9 is "as good as longer clinicianadministered instruments" for the screening of MDD. ${ }^{16}$ Ultimately, the PHQ-9 is the most thoroughly evaluated screening tool for $\mathrm{MDD},{ }^{17}$ and appears to be an ideal option when initiating screening for MDD in the otolaryngology clinic.

There are several caveats that must be considered when interpreting the results of the present study. The response rate in the present study was low, at $7 \%$. Prior survey studies targeting the ARS have recorded response rates ranging between $7 \%$ and $32 \% .{ }^{18}$ A possible factor in the low response rate for the current study may be respondent fatigue. This is a known phenomenon that usually occurs toward the tail end of a survey; as the respondent loses interest or focus, he/ she may begin skipping questions or selecting responses in a random fashion. ${ }^{19}$ Furthermore, MDD may be of less clinical interest to otolaryngologists, limiting survey responses. We attempted, in part, to counteract this phenomenon by targeting a study group that would be most interested in the topic at hand - that is, members of the ARS. Although this can lead to non-response bias, as respondents with a vested interest in comorbid depression and CRS may be more likely to evaluate or treat depression, these data suggest the opposite. Despite this response bias, very few providers screen for and treat depression. This degree of unexpected homogeneity in the response of the group is certainly striking and serves to highlight a contradiction between "belief" and "action." Furthermore, the overall modest sample size and the occurrence of incomplete responses to certain questions may also have limited the sensitivity of the chi-squared analysis. Given the limited data on practice patterns of otolaryngologists in the diagnosis and management of comorbid MDD in patients with CRS, the present study presents a valuable step toward improving the complete care of the CRS patient by understanding physician attitudes.

\section{Conclusion}

Formal training in the diagnosis and management of MDD is rare in rhinology practices. Most ARS members believe that comorbid MDD impacts patient outcomes, although a large proportion of surgeons neither screen nor feel comfortable diagnosing MDD.

\section{Conflict of Interests}

The authors have no conflict of interests to declare.

\section{References}

1 Zhou S, Hur K, Shen J, Wrobel B. Impact of sinonasal disease on depression, sleep duration, and productivity among adults in the United States. Laryngoscope Investig Otolaryngol 2017;2(05): 288-294

2 Litvack JR, Mace J, Smith TL. Role of depression in outcomes of endoscopic sinus surgery. Otolaryngol Head Neck Surg 2011;144 (03):446-451

3 Davis GE, Yueh B, Walker E, Katon W, Koepsell TD, Weymuller EA. Psychiatric distress amplifies symptoms after surgery for chronic rhinosinusitis. Otolaryngol Head Neck Surg 2005;132(02):189196

4 Schlosser RJ, Gage SE, Kohli P, Soler ZM. Burden of illness: A systematic review of depression in chronic rhinosinusitis. Am J Rhinol Allergy 2016;30(04):250-256 
5 Schlosser RJ, Hyer JM, Smith TL, et al. Depression-Specific Outcomes After Treatment of Chronic Rhinosinusitis. JAMA Otolaryngol Head Neck Surg 2016;142(04):370-376

6 Schlosser RJ, Storck K, Cortese BM, Uhde TW, Rudmik L, Soler ZM. Depression in chronic rhinosinusitis: A controlled cohort study. Am J Rhinol Allergy 2016;30(02):128-133

7 Erskine SE, Hopkins C, Clark A, et al. Chronic rhinosinusitis and mood disturbance. Rhinology 2017;55(02):113-119

8 Wasan A, Fernandez E, Jamison RN, Bhattacharyya N. Association of anxiety and depression with reported disease severity in patients undergoing evaluation for chronic rhinosinusitis. Ann Otol Rhinol Laryngol 2007;116(07):491-497

9 Hanna BM, Crump RT, Liu G, Sutherland JM, Janjua AS. Incidence and burden of comorbid pain and depression in patients with chronic rhinosinusitis awaiting endoscopic sinus surgery in Canada. J Otolaryngol Head Neck Surg 2017;46(01):23

10 Tomoum MO, Klattcromwell C, DelSignore A, Ebert C, Senior BA. Depression and anxiety in chronic rhinosinusitis. Int Forum Allergy Rhinol 2015;5(08):674-681

11 Cox DR, Ashby S, DeConde AS, et al. Dyad of pain and depression in chronic rhinosinusitis. Int Forum Allergy Rhinol 2016;6(03):308-314

12 Young AK, Young BK, Riley LH III, Skolasky RL. Assessment of presurgical psychological screening in patients undergoing spine surgery: use and clinical impact. J Spinal Disord Tech 2014;27 (02):76-79
13 Goldin Evans M, Phillippi S, Gee RE. Examining the Screening Practices of Physicians for Postpartum Depression: Implications for Improving Health Outcomes. Womens Health Issues 2015;25 (06):703-710

14 Adams KN, Schuman TA, Ebert CS, You W, Tomoum MO, Senior BA. Self-reported anxiety and depression unchanged after endoscopic sinus surgery for chronic rhinosinusitis. Rhinology 2018;56(03): 234-240

15 Banoub RG, Hoehle LP, Phillips KM, et al. Depressed Mood Modulates Impact of Chronic Rhinosinusitis Symptoms on Quality of Life. J Allergy Clin Immunol Pract 2018

16 Gilbody S, Richards D, Brealey S, Hewitt C. Screening for depression in medical settings with the Patient Health Questionnaire (PHQ): a diagnostic meta-analysis. J Gen Intern Med 2007;22(11): 1596-1602

17 El-Den S, Chen TF, Gan YL, Wong E, O'Reilly CL. The psychometric properties of depression screening tools in primary healthcare settings: A systematic review. J Affect Disord 2018;225: 503-522

18 Chen S, Le CH, Liang J. Practice patterns in endoscopic dacryocystorhinostomy: survey of the American Rhinologic Society. Int Forum Allergy Rhinol 2016;6(09):990-997

19 Respondent Fatigue PB-N. In: PJ L, ed. Encyclopedia of Survey Research Methods. 2008:742-743 